\title{
Risk Factors for Anastomotic Leakage after Colorectal Surgery with Double-staple Technique Anastomosis: Impact of the Agatston Score
}

\author{
Yosuke Namba $^{1)}$, Shoichiro Mukai ${ }^{1)}$, Yasufumi Saito ${ }^{1)}$, Toshiyuki Moriuchi ${ }^{1)}$, Tomoaki Bekki ${ }^{1)}$, Sho Okimoto ${ }^{1)}$, Koichi Oishi ${ }^{1)}$, \\ Seiji Fujisaki ${ }^{1}$, Mamoru Takahashi ${ }^{1}$, Toshikatsu Fukuda ${ }^{1)}$, Hiroyuki Egi' ${ }^{2)}$ and Hideki Ohdan ${ }^{2)}$ \\ 1) Department of Surgery, Chugoku Rosai Hospital, Hiroshima, Japan \\ 2) Department of Gastroenterological and Transplant Surgery Applied Life Sciences, Institute of Biomedical and Health \\ Sciences, Hiroshima University, Hiroshima, Japan
}

\begin{abstract}
Objectives: Anastomotic leakage is associated with severe morbidity, mortality, and functional defects. Its risk factors remain unclear. However, blood perfusion may be a potential major risk factor. It has been reported that the Agatston score is an index for blood flow perfusion evaluation. Therefore, we evaluated the clinical indicators associated with anastomotic leakage, including the Agatston score, in patients who underwent colorectal surgery.

Methods: We retrospectively analyzed 147 patients who underwent elective colorectal surgery with the double-staple technique anastomosis for colorectal cancer between April 2015 and March 2020. The primary outcome was the presence or absence of anastomotic leakage. Univariate and multivariate analyses were employed to identify pre- and intraoperative risk factors.

Results: Of the 147 patients analyzed, anastomotic leakage occurred in 12 (8.16\%). Male gender, history of angina and myocardial infarction, preoperative white blood cell count, the Agatston score, extent of bleeding, operation time, and intraoperative fluid volume were significantly related to a higher incidence of anastomotic leakage in univariate analysis. Multivariate analysis demonstrated that the incidence of anastomotic leakage was high in patients with a high Agatston score.

Conclusions: The Agatston score can predict the incidence of anastomotic leakage in patients following colorectal surgery. Thus, perioperative measures to prevent anastomotic leakage are recommended when a high Agatston score is observed. A prospective trial is required to demonstrate, with a high level of evidence, that the Agatston score can be useful as a risk score for anastomotic leakage following colorectal surgery.
\end{abstract}

\section{Keywords}

Agatston score, anastomotic leakage, colorectal surgery, double stapling technique

J Anus Rectum Colon 2021; 5(2): 181-187

\section{Introduction}

Postoperative anastomotic leakage (AL) is a common and major complication of colorectal surgery. It is associated with functional defects as well as severe morbidity and mor- tality[1-3]. In addition, AL is associated with the risk of local cancer recurrence, which reduces the rate of overall and disease-free survival[4-6]. Although the double-staple technique (DST) has significantly facilitated bowel reconstruction following colorectal surgery, the incidence of postopera- 
tive $\mathrm{AL}$ is $6.3 \%-13.7 \%$. While postoperative $\mathrm{AL}$ is linked to multiple factors, including the surgical technique, tumor location, sex, and intestinal bacterial infection[7-11], the exact risk factors for AL remain unclear. Several risk factor analyses for AL following colorectal surgery have been conducted. However, accurate evaluation of the risk of $\mathrm{AL}$ in patients is difficult $[3,12,13]$.

Blood perfusion is one of the major surgical risk factors for AL[14,15]. Ris et al. reported that assessment of anastomotic blood perfusion via the indocyanine green assay helped prevent AL[16]. Conversely, Du et al. reported that measurement of the anastomotic colon using Doppler sonographic hemodynamics was effective against AL[17].

The Agatston score is an index for the evaluation of blood flow perfusion and is utilized to assess the degree of arteriosclerosis. It indicates the amount of calcium in the artery; moreover, it is estimated via computed tomography (CT) and expressed as a numerical value. Owing to its ability to detect calcium in the tissues, CT can quantify the amount of calcium in blood vessels, which represents the plaque burden and is associated with the progression of atherosclerosis[18,19]. The Agatston score is the most evaluated score in routine clinical investigations for the objective assessment of coronary artery calcium[20]. We speculated that it could be used to evaluate blood perfusion in intestinal anastomosis.

This study aimed to assess the clinical indicators (including the Agatston score) associated with AL in patients who underwent elective colorectal surgery for colorectal cancer with DST anastomosis.

\section{Methods}

\section{Study population}

This single center retrospective cohort study evaluated 150 colorectal cancer patients who underwent elective colorectal surgery with bowel resection and DST anastomosis between April 2015 and March 2020 at our hospital. Of these patients, three who were not examined via noncontrast CT were excluded. Therefore, 147 patients were included for further analyses. Among these patients, AL was observed in $12(8.16 \%)$, who were categorized into the "AL group," whereas the remaining 135 patients were categorized into the "No-AL group."

The patients' baseline demographics, clinical characteristics, and perioperative factors were evaluated as the risk factors for $\mathrm{AL}$ and compared between the $\mathrm{AL}$ and No-AL groups. The baseline demographics included age, sex, body weight, body mass index, American Society of Anesthesiologists score, Eastern Cooperative Oncology Group/World Health Organization Performance Status, history of angina, myocardial infarction and cerebrovascular disease, diabetes, and the Brinkman index. The clinical characteristics in- cluded tumor location, depth of invasion, nodal status, TNM stage, preoperative intestinal decompression, Agatston score, and preoperative blood test results. The perioperative factors included the extent of bleeding, operation time, intraoperative fluid volume, type of surgery (open or laparoscopic), presence of protective diverting stoma, distance of anastomosis from the anal verge, number of linear staplers, size of the circular stapler, and preservation or dissection of the left colic artery. The TNM stage was classified using the Union for International Cancer Control TNM Classification of Malignant Tumors, 8th edition. Intestinal decompression was defined as the placement of an ileus tube or stent.

Patients provided their informed consent for the use of their data. The study was approved by the Medical Ethics Committee of Chugoku Rosai Hospital (Approval No. 202021).

\section{Agatston score calculation procedure}

The Agatston score was calculated using non-contrast CT with a slice thickness of $5 \mathrm{~mm}$ and a dedicated software (AW Server, GE Healthcare, Japan). The amount of calcium in the aorta from the bifurcation of the renal arteries to the bifurcation of the common iliac arteries was calculated (Figure 1a and 1b). The Agatston score was calculated by two surgeons in all patients, and the average value was considered for final analysis.

\section{Definition of anastomotic leakage}

$\mathrm{AL}$ was defined as a symptomatic leak diagnosed and managed within 30 days of the primary resection. The severity of the leakage was classified according to the ClavienDindo classification, and a grade of II or higher was defined as AL.

\section{Statistical analysis}

Data were analyzed using the Statistical Package for the Social Sciences (SPSS) version 22.0 (SPSS Inc., Chicago, IL, USA). Descriptive statistics for categorical and continuous variables were reported as absolute numbers and mean \pm standard deviation, respectively. Categorical variables were analyzed using Fisher's exact test, whereas continuous variables were analyzed using the Student's $t$-test. Statistical significance was set to $P<0.05$. For continuous variables with $P<0.05$, as indicated by univariate analysis, cutoff values were set using the receiver operating characteristic curve analysis. Multivariate logistic regression analysis was conducted using factors with $P<0.05$ in the univariate analysis as the independent variables and the presence or absence of $\mathrm{AL}$ as the dependent variable. For independent variables that were expected to exhibit strong correlations with the presence or absence of $\mathrm{AL}$, internal correlations were calculated using Spearman's rank correlation test to avoid multicollinearity. If the correlation was very strong, one of the fac- 

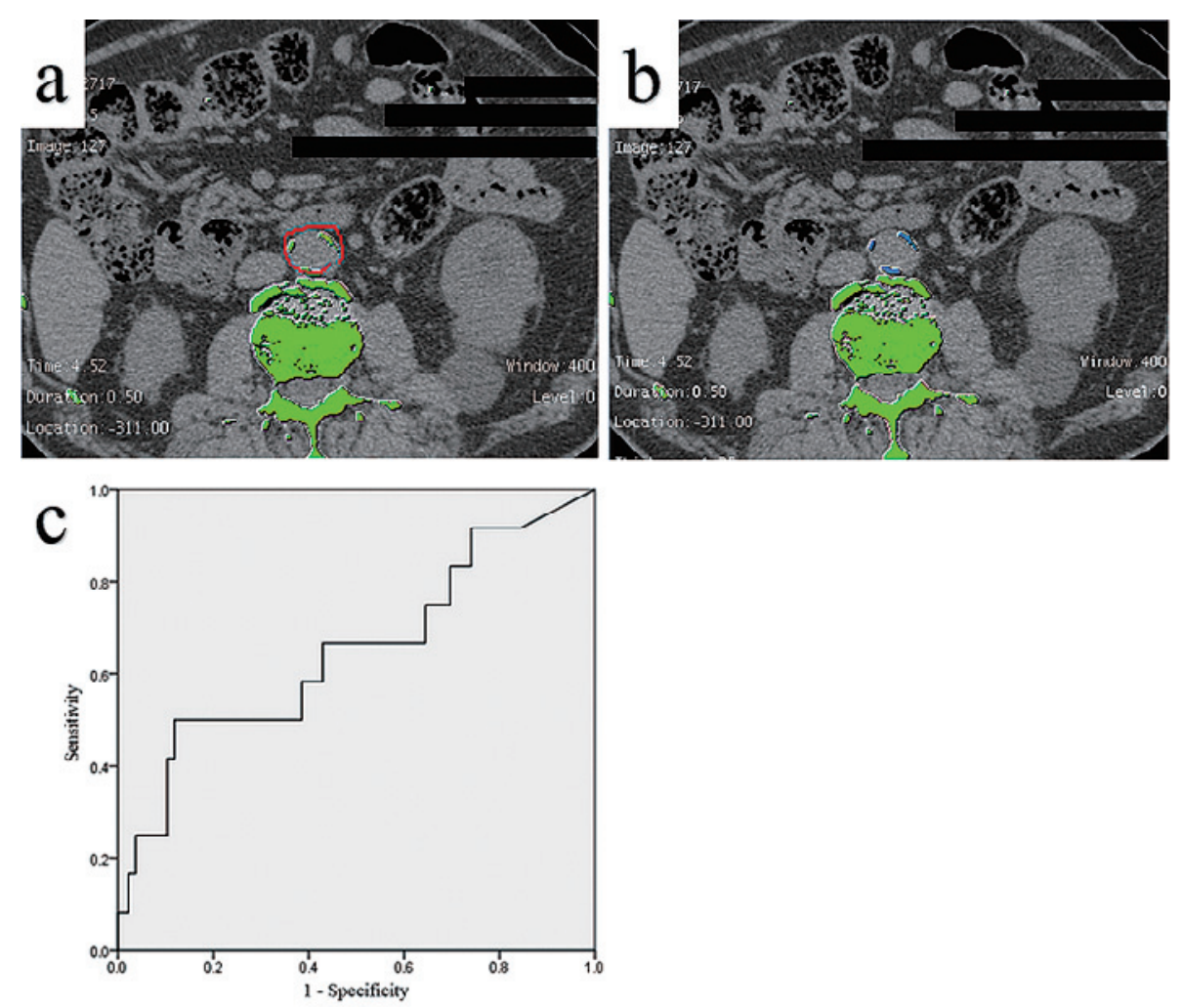

Figure 1. The Agaston score measurement screen of computed tomography (CT) scan and receiver operating characteristic curves for the Agatston score.

We calculated the amount of calcium in the aorta from the bifurcation of the renal arteries to the bifurcation of the common iliac arteries using non-contrast CT and a dedicated software (AW Server, GE Healthcare, Japan). (a) The calcified area in the aorta was selected. (b) The selected area turned blue. The calcified area of each slice was quantified. (c) The area under the curve (AUC) is 0.65 (95\% CI, 0.45-0.84), and the cutoff value is 4007 (sensitivity: 0.50 , specificity: 0.88$)$.

tors was excluded. Lower rectal tumor and the distance of anastomosis from the anal verge, which are known as risk factors for AL[21,22], were equally included in the multivariate logistic regression analysis.

\section{Results}

The patients' baseline demographics and clinical characteristics are presented in Table 1, 2, respectively. The Clavien-Dindo grades of AL were II and III in $41.7 \%$ (5/12) and $58.3 \%(7 / 12)$ of the patients in the AL group, respectively. The percentage of males, history of angina and myocardial infarction, preoperative WBC count, and Agatston score differed between the No-AL and AL groups (55.5\% vs. $91.6 \%[P=0.01], 11.8 \%$ vs. $33.3 \%[P=0.04,6,440.0 \pm$ $2,077.9 \times 10^{3} / \mu \mathrm{L}$ vs. $8,095.8 \pm 4,383.5 \times 10^{3} / \mu \mathrm{L}[P=0.01]$, and $3217.5 \pm 3279.7$ vs. $1476.8 \pm 1849.1[P<0.01]$, respectively).

Table 3 presents the surgical characteristics analyzed via univariate analysis. The extent of bleeding, operation time, and intraoperative fluid volume significantly differed between the No-AL and AL groups $(187.3 \pm 360.1 \mathrm{~mL}$ vs. $504.1 \pm 1,234.6 \mathrm{~mL}[P=0.03], 324.7 \pm 105.1 \mathrm{~min}$ vs. $406.7 \pm 128.6 \mathrm{~min}[P=0.01]$, and $2,860.9 \pm 824.0 \mathrm{~mL}$ vs. $3,097.5 \pm 1,816.1 \mathrm{~mL}[P=0.01]$, respectively).

Internal correlations were calculated between the history of angina/myocardial infarction and the Agatston score, as well as among the extent of bleeding, operation time, and intraoperative fluid volume, all of which were speculated to have a strong clinical correlation with the presence or absence of AL. Accordingly, history of angina/myocardial infarction, extent of bleeding, and operation time were excluded from the multivariate logistic regression analysis to avoid multicollinearity, as strong correlations were observed between history of angina/myocardial infarction and the Agatston score $(\mathrm{r}=0.30, P<0.01)$, extent of bleeding and operation time $(\mathrm{r}=0.42, P<0.01)$, extent of bleeding and intraoperative fluid volume $(\mathrm{r}=0.49, P<0.01)$, and operation time and intraoperative fluid volume $(\mathrm{r}=0.60, P<$ $0.01)$. 
Table 1. Baseline Demographics.

\begin{tabular}{lccc}
\hline & No-AL group & AL group & \\
& $\mathrm{n}=135(91.8 \%)$ & $\mathrm{n}=12(8.16 \%)$ & $p$ value \\
\hline Age, mean (SD), $\mathrm{y}$ & $73.4 \pm 9.96$ & $70.4 \pm 10.4$ & 0.31 \\
Male gender, $\mathrm{n}(\%)$ & $75(55.5)$ & $11(91.6)$ & 0.01 \\
Body weight, mean (SD), $\mathrm{kg}$ & $59.3 \pm 12.5$ & $59.2 \pm 8.03$ & 0.98 \\
Body mass index, mean (SD), kg/m ${ }^{2}$ & $23.9 \pm 5.50$ & $23.3 \pm 3.76$ & 0.72 \\
ASA score, $\mathrm{n}(\%)$ & & & 0.44 \\
$\mathrm{I}$ & $8(5.90)$ & $1(8.30)$ & \\
II & $103(76.2)$ & $8(66.6)$ & \\
III & $24(17.7)$ & $3(25.0)$ & \\
Performance status (2-4), n (\%) & $14(10.3)$ & $3(25.0)$ & 0.14 \\
Brinkman index > 400, $\mathrm{n}(\%)$ & $44(33.5)$ & $5(31.2)$ & 1 \\
History of cerebrovascular disease, $\mathrm{n}(\%)$ & $13(9.62)$ & $3(25.0)$ & 0.12 \\
History of angina or myocardial infarction, $\mathrm{n}(\%)$ & $16(11.8)$ & $4(33.3)$ & 0.04 \\
Diabetes, $\mathrm{n}(\%)$ & $28(20.7)$ & $2(16.6)$ & 1 \\
\hline
\end{tabular}

Sex, performance status, history of cerebrovascular disease, angina or myocardial infarction, diabetes, and Brinkman index $>400$ were analyzed using Fisher's exact test, and other factors were analyzed using Student's $t$-test.

ASA, American Society of Anesthesiologists; AL, anastomotic leakage; SD, standard deviation

Table 2. Clinical Characteristics.

\begin{tabular}{|c|c|c|c|}
\hline & $\begin{array}{c}\text { No-AL group } \\
\mathrm{n}=135(91.8 \%)\end{array}$ & $\begin{array}{c}\text { AL group } \\
\mathrm{n}=12(8.16 \%)\end{array}$ & $p$ value \\
\hline Tumor location, n (\%) & & & 0.35 \\
\hline Sigmoid colon & $76(56.2)$ & $5(41.6)$ & \\
\hline Rectosigmoid junction & $32(23.7)$ & $3(25.0)$ & \\
\hline Upper rectum & $19(14.0)$ & $2(16.6)$ & \\
\hline Lower rectum & $8(5.90)$ & $2(16.6)$ & \\
\hline Depth of invasion, $\mathrm{n}(\%)$ & & & 0.52 \\
\hline $\mathrm{T} 1$ & $19(14.0)$ & $0(0)$ & \\
\hline $\mathrm{T} 2$ & $12(8.88)$ & $1(8.30)$ & \\
\hline $\mathrm{T} 3$ & $73(54.0)$ & $9(75.0)$ & \\
\hline $\mathrm{T} 4$ & $32(22.9)$ & $2(16.6)$ & \\
\hline Nodal status, n (\%) & & & 0.90 \\
\hline N0 & $90(66.6)$ & $8(66.6)$ & \\
\hline N1 & $34(25.1)$ & $4(33.3)$ & \\
\hline N2 & $11(8.14)$ & $0(0)$ & \\
\hline TNM stage, n (\%) & & & 0.91 \\
\hline I & $24(17.7)$ & $1(8.30)$ & \\
\hline II & $63(46.6)$ & $7(58.3)$ & \\
\hline III & $34(25.1)$ & $3(25.0)$ & \\
\hline IV & $13(9.62)$ & $1(8.30)$ & \\
\hline Preoperative intestinal decompression, n (\%) & $32(22.9)$ & $4(33.3)$ & 0.48 \\
\hline Blood sugar level, mean (SD), mg/dL & $122.8 \pm 42.8$ & $110.3 \pm 18.3$ & 0.24 \\
\hline Preop WBC count, mean (SD), / $\mu \mathrm{L}$ & $6440.0 \pm 2077.9$ & $8095.8 \pm 4383.5$ & 0.01 \\
\hline Preop CRP, mean (SD), mg/dL & $1.01 \pm 2.44$ & $2.13 \pm 4.22$ & 0.16 \\
\hline Preop albumin, mean (SD), g/dL & $3.79 \pm 0.64$ & $3.73 \pm 0.77$ & 0.70 \\
\hline Preop eGFR, mean (SD), mL/minutes $/ 1.73 \mathrm{~m}^{2}$ & $71.1 \pm 22.7$ & $76.8 \pm 18.4$ & 0.35 \\
\hline Agatston score, mean (SD) & $1476.8 \pm 1849.1$ & $3217.5 \pm 3279.7$ & $<0.01$ \\
\hline
\end{tabular}

Tumor location, depth of invasion, nodal status, TNM stage, and preoperative intestinal decompression were analyzed using Fisher's exact test, and other factors were analyzed using Student's $t$-test.

AL, anastomotic leakage; Preop, preoperative; WBC, white blood cell; CRP, C-reactive protein; eGFR, estimated glomerular filtration rate; SD, standard deviation 
Table 3. Surgical Characteristics.

\begin{tabular}{lccc}
\hline & No-AL group & AL group & $p$ value \\
& $\mathrm{n}=135(91.8 \%)$ & $\mathrm{n}=12(8.16 \%)$ & \\
\hline Extent of bleeding, mean (SD), $\mathrm{mL}$ & $187.3 \pm 360.1$ & $504.1 \pm 1234.6$ & 0.03 \\
Operation time, mean (SD), minutes & $324.7 \pm 105.1$ & $406.7 \pm 128.6$ & 0.01 \\
Intraoperative fluid volume, mean (SD), $\mathrm{mL}$ & $2360.9 \pm 824.0$ & $3097.5 \pm 1816.1$ & 0.01 \\
Laparoscopic surgery, $\mathrm{n}(\%)$ & $108(80.0)$ & $9(75.0)$ & 0.71 \\
Protective diverting stoma, $\mathrm{n}(\%)$ & $28(20.7)$ & $3(25.0)$ & 0.71 \\
Preservation of the LCA, $\mathrm{n}(\%)$ & $69(51.1)$ & $7(58.3)$ & 0.76 \\
Distance of anastomosis from the anal verge, mean (SD), cm & $10.0 \pm 3.7$ & $10.1 \pm 6.1$ & 0.89 \\
Size of the circular stapler & & & 0.76 \\
25 mm, n (\%) & $79(58.5)$ & $6(50.0)$ & \\
29 mm, $\mathrm{n}(\%)$ & $56(41.5)$ & $6(50.0)$ & \\
Number of linear staplers & & & 0.27 \\
$1, \mathrm{n}(\%)$ & $114(84.4)$ & $10(83.3)$ & \\
$2, \mathrm{n}(\%)$ & $19(14.0)$ & $1(8.3)$ & \\
$3, \mathrm{n}(\%)$ & $2(1.4)$ & $1(8.3)$ & \\
\hline
\end{tabular}

Laparotomy, protective diverting stoma, preservation of the LCA, and number of linear staplers were analyzed using Fisher's exact test, and other factors were analyzed using Student's $t$-test.

$\mathrm{AL}$, anastomotic leakage; LCA, left colic artery; SD, standard deviation

Table 4. Multivariate Logistic Regression with Clinical and Surgical Indicators.

\begin{tabular}{lcc}
\hline & Odds Ratio $(95 \% \mathrm{CI})$ & $p$ value \\
\hline Male gender & $7.40(0.86-63.0)$ & 0.06 \\
Lower rectal tumor & $2.82(0.32-24.5)$ & 0.34 \\
Preop WBC count & $1.37(0.31-6.02)$ & 0.67 \\
Agatston score & $6.09(1.48-25.0)$ & 0.01 \\
Intraoperative fluid volume & $2.90(0.65-12.8)$ & 0.15 \\
Distance of anastomosis from the anal verge & $0.66(0.14-2.99)$ & 0.59 \\
\hline
\end{tabular}

Preop, preoperative; WBC, white blood cell

The cutoff values were set for the lower rectal tumor, distance of anastomosis from the anal verge, and continuous variables that significantly differed between the two groups. In addition, multivariate logistic regression analysis was conducted. The multivariate logistic regression analysis revealed that the Agatston score was independently associated with the development of $\mathrm{AL}$ (odds ratio $[\mathrm{OR}]=6.09,95 \%$ confidence interval [CI] 1.48-25.0]; $P=0.01$ ) (Table 4). The cutoff value of the Agatston score was estimated to be 4007 (sensitivity: 0.50 , specificity: 0.88 , area under the curve (AUC): 0.65) (Figure 1c).

\section{Discussion}

This study revealed that the Agatston score was a factor that predicted the incidence of AL after an elective colorectal surgery with the DST for colon cancer. AL is a major problem in patients who underwent colorectal surgery. It is associated with postoperative morbidity and mortality, local recurrence, and worse patient survival[23,24]. Several risk factors, including age, sex, obesity, intraoperative bleeding, and protective diverting stoma, have been reported to be associated with AL following colorectal surgery $[9,25,26]$.

Tension-free anastomosis with adequate blood perfusion is important for the prevention of AL[15,27]. Several methods are available to determine blood flow and oxygenation, which include the assessment of anastomotic blood perfusion using the indocyanine green assay and measurement of the anastomotic blood flow via Doppler sonography[28]. While these are useful for evaluating intraoperative blood perfusion, no method for evaluating preoperative blood perfusion has been developed. Although it was reported that the Agatston score is associated with coronary events[29], we speculated that it is also associated with anastomotic blood perfusion in colorectal surgery. The Agatston score is an index for the quantification of the artery calcium levels using non-contrast $\mathrm{CT}$ and the most evaluated score in routine clinical investigations for the objective assessment of coronary artery calcium[20,30]. Because the calcification of the central blood vessels reflects that of the peripheral blood 
vessels, calcification from the bifurcation of the renal arteries to the bifurcation of the common iliac arteries is considered to be involved in the decrease of intestinal blood perfusion and AL. To the best of our knowledge, this study is the first to demonstrate that the Agatston score is associated with the development of AL following colorectal surgery with DST for colon cancer. The Agatston score was not significantly associated with postoperative defecation function, other complications, or degree of complications.

The finding that the Agatston score was associated with AL following colorectal surgery with DST makes sense from the perspective of blood perfusion and is a valuable finding as a preoperative predictor of AL. If a high Agatston score is identified during management, strict follow-up involving indwelling intraperitoneal drain for a long period of time, increasing preparation intensity, or delaying the start of oral intake is recommended. Although this study did not include the indocyanine green assay or Doppler ultrasonography for the assessment of anastomotic blood perfusion, the combined use of these intraoperative assessment methods and the preoperative assessment method based on the Agatston score is considered to lead to a high prediction rate of AL.

The present study has several limitations. First, this study was conducted at a single center and was retrospective in design. Therefore, selection bias cannot be ruled out. Second, the Agatston score is computed through a manual selection of the calcification for measurements, and this can vary depending on the investigator. Finally, this study did not evaluate intraoperative blood flow perfusion. We plan to increase the number of patients in the future and evaluate the relationship between intraoperative blood flow using indocyanine green and preoperative blood flow using the Agatston score. Within the limitations of this study, we consider that the Agatston score is associated with the development of AL. Further studies with larger cohorts of patients will be important to provide additional support to these findings.

In conclusion, our results indicate that the Agatston score can predict the incidence of AL. Perioperative management to prevent $\mathrm{AL}$ is recommended when these factors are observed. Moreover, a prospective trial is required to demonstrate, with a high level of evidence, that the Agatston score can be useful as a risk score for AL following colorectal surgery.

\section{Conflicts of Interest}

There are no conflicts of interest.

\section{Author Contributions}

All authors contributed to the study conception and design. Material preparation, data collection and analysis were performed by YN and SM. The first draft of the manuscript was written by $\mathrm{YN}$ and all authors commented on previous versions of the manuscript. SM read and approved the final manuscript. TF provided clinical advice, and HO supervised the report.

Approval by Institutional Review Board (IRB)

The study was approved by the Medical Ethics Committee of Chugoku Rosai Hospital (No. 2020-21).

\section{References}

1. Walker KG, Bell SW, Rickard MJ, et al. Anastomotic leakage is predictive of diminished survival after potentially curative resection for colorectal cancer. Ann Surg. 2004 Aug; 240(2): 255-9.

2. Bell SW, Walker KG, Rickard MJ, et al. Anastomotic leakage after curative anterior resection results in a higher prevalence of local recurrence. Br J Surg. 2003 Oct; 90(10): 1261-6.

3. Penna M, Hompes R, Arnold $\mathrm{S}$, et al. Incidence and risk factors for anastomotic failure in 1594 patients treated by transanal total mesorectal excision: results from the International TaTME registry. Ann Surg. 2019 Apr; 269(4): 700-11.

4. Nachiappan S, Askari A, Malietzis G, et al. The impact of anastomotic leak and its treatment on cancer recurrence and survival following elective colorectal cancer resection. World J Surg. 2015 Apr; 39(4): 1052-8.

5. Boccola MA, Buettner PG, Rozen WM, et al. Risk factors and outcomes for anastomotic leakage in colorectal surgery: a singleinstitution analysis of 1576 patients. World J Surg. 2011 Jan; 35 (1): 186-95.

6. Khoury W, Lavery LC, Kiran RP. Impact of early reoperation after resection for colorectal cancer on long-term oncological outcomes. Colorectal Dis. 2012 Mar; 14(3): e117-23.

7. Phitayakorn R, Delaney CP, Reynolds HL, et al. Standardized algorithms for management of anastomotic leaks and related abdominal and pelvic abscesses after colorectal surgery. World $\mathrm{J}$ Surg. 2008 Jun; 32(6): 1147-56.

8. Paun BC, Cassie S, MacLean AR, et al. Postoperative complications following surgery for rectal cancer. Ann Surg. 2010 May; 251(5): 807-18.

9. Jung SH, Yu CS, Choi PW, et al. Risk factors and oncologic impact of anastomotic leakage after rectal cancer surgery. Dis Colon Rectum. 2008 Jun; 51(6): 902-8.

10. Boström $P$, Haapamäk MM, Rutegård $J$, et al. Population-based cohort study of the impact on postoperative mortality of anastomotic leakage after anterior resection for rectal cancer. BJS Open. 2019 Oct; 3(1): 106-11.

11. Kang CY, Halabi WJ, Chaudhry OO, et al. Risk factors for anastomotic leakage after anterior resection for rectal cancer. JAMA Surg. 2013 Jan; 148(1): 65-71.

12. Tanaka K, Okuda J, Yamamoto $S$, et al. Risk factors for anastomotic leakage after laparoscopic surgery with the double stapling technique for stage 0/I rectal carcinoma: a subgroup analysis of a multicenter, single-arm phase II trial. Surg Today. 2017 Oct; 47 (10): 1215-22.

13. Su'a BU, Mikaere HL, Rahiri JL, et al. Systematic review of the role of biomarkers in diagnosing anastomotic leakage following colorectal surgery. Br J Surg. 2017 Apr; 104(5): 503-12.

14. Morse BC, Simpson JP, Jones YR, et al. Determination of independent predictive factors for anastomotic leak: analysis of 682 in- 
testinal anastomoses. Am J Surg. 2013 Dec; 206(6): 950-5.

15. Rutegård M, Rutegård J. Anastomotic leakage in rectal cancer surgery: the role of blood perfusion. World J Gastrointest Surg. 2015 Nov; 7(11): 289-92.

16. Ris F, Liot E, Buchs NC, et al. Multicentre phase II trial of nearinfrared imaging in elective colorectal surgery. Br J Surg. 2018 Sep; 105(10): 1359-67.

17. Du CZ, Fan ZH, Yang YF, et al. Value of intra-operative Doppler sonographic measurements in predicting post-operative anastomotic leakage in rectal cancer: a prospective pilot study. Chin Med J. 2019 Sep; 132(18): 2168-76.

18. Xu Y, Mintz GS, Tam A, et al. Prevalence, distribution, predictors, and outcomes of patients with calcified nodules in native coronary arteries: a 3-vessel intravascular ultrasound analysis from providing Regional Observations to Study Predictors of Events in the Coronary Tree (PROSPECT). Circulation. 2012 Jul; 126(5): 53745 .

19. Vliegenthart R, Morris PB. Computed tomography coronary artery calcium scoring: review of evidence base and cost-effectiveness in cardiovascular risk prediction. J Thorac Imaging. 2012 Sep; 27(5): 296-303.

20. Detrano R, Guerci AD, Carr JJ, et al. Coronary calcium as a predictor of coronary events in four racial or ethnic groups. N Engl J Med. 2008 Mar; 358(13): 1336-45.

21. Kim CH, Lee SY, Kim HR, et al. Nomogram prediction of anastomotic leakage and determination of an effective surgical strategy for reducing anastomotic leakage after laparoscopic rectal Cancer surgery. Gastroenterol Res Pract. 2017; 2017: 4510561.

22. Lee $S$, Ahn B, Lee $S$. The relationship between the number of intersections of staple lines and anastomotic leakage after the use of a double stapling technique in laparoscopic colorectal Surgery. Surg Laparosc Endosc Percutan Tech. 2017 Aug; 27(4): 273-81.
23. den Dulk M, Marijnen CA, Collette L, et al. Multicentre analysis of oncological and survival outcomes following anastomotic leakage after rectal cancer surgery. Br J Surg. 2009 Sep; 96(9): 106675 .

24. Mirnezami A, Mirnezami R, Chandrakumaran K, et al. Increased local recurrence and reduced survival from colorectal cancer following anastomotic leak: systematic review and meta-analysis. Ann Surg. 2011 May; 253(5): 890-9.

25. Jestin P, Påhlman L, Gunnarsson U. Risk factors for anastomotic leakage after rectal cancer surgery: a case-control study. Colorectal. Dis. 2008 Sep; 10(7): 715-21.

26. Yeh CY, Changchien CR, Wang JY, et al. Pelvic drainage and other risk factors for leakage after elective anterior resection in rectal cancer patients: a prospective study of 978 patients. Ann Surg. 2005 Jan; 241(1): 9-13.

27. Yasuda $\mathrm{K}$, Kawai $\mathrm{K}$, Ishihara $\mathrm{S}$, et al. Level of arterial ligation in sigmoid colon and rectal cancer surgery. World J Surg Oncol. 2016 Apr; 14: 99.

28. Urbanavičius L, Pattyn P, de Putte DV, et al. How to assess intestinal viability during surgery: A review of techniques. World $\mathrm{J}$ Gastrointest Surg, 2011 May; 3(5): 59-69.

29. Dudink E, Peeters F, Altintas S, et al. Agatston score of the descending aorta is independently associated with coronary events in a low-risk population. Open Heart. 2018 Nov; 5(2): e000893.

30. Agatston AS, Janowitz WR, Hildner FJ, et al. Quantification of coronary calcified artery calcium using ultrafast computed tomography. J Am Coll Cardiol. 1990 Mar; 15(4): 827-32.

Journal of the Anus, Rectum and Colon is an Open Access journal distributed under the Creative Commons Attribution-NonCommercial-NoDerivatives $4.0 \mathrm{In}$ ternational License. To view the details of this license, please visit (https://creativ ecommons.org/licenses/by-nc-nd/4.0/). 